\title{
A GeoSocial Intelligence Framework for Studying \& Promoting Resilience to Seasonal Flooding in Jakarta, Indonesia
}

\author{
Dr. Tomas Holderness, Dr. Etienne Turpin and Dr. Rohan Wickramasuriya \\ SMART Infrastructure Facility, University of Wollongong, Australia
}

\section{PAPER ABSTRACT / BRIEF}

PetaJakarta.org is a web-based platform developed to harness the power of social media to gather, sort, and display information about flooding for Jakarta residents in real time. The platform runs on the open source software CogniCity-an OSS platform developed by the SMART Infrastructure Facility, University of Wollongong - which allows data to be collected and disseminated by community members through their location-enabled mobile devices. The project uses a GeoSocial Intelligence Framework to approach the complexity of Jakarta's entangled hydraulic, hydrological and meteorological systems and thereby converts the noise of social media into knowledge about urban infrastructure and situational conditions related to flooding and inundation.

In this paper, PetaJakarta.org co-directors Dr Tomas Holderness, Geomatics Research Fellow at the SMART Infrastructure Facility, Dr Etienne Turpin, Vice-Chancellor's Postdoctoral Research Fellow at the SMART Infrastructure Facility, and Dr Rohan Wickramasuriyam, GIS Research Fellow at the SMART Infrastructure Facility, will discuss their GeoSocial Intelligence Framework as it applies to their current research in Jakarta. They will also present their preliminary findings from their 2014 Twitter \#DataGrant, which has allowed them to develop a correlative analysis between historic social media information, the Jakarta government's flood maps, and the infrastructure used to manage critical flood emergencies. Finally, they will speculate on several future applications of the CogniCity OSS and suggest how it might be developed to further promote an integrated civic co-management platform with the support of business, industry, government and community organizations.

Keywords: Urban Resilience, Crowdsourcing, Social Media, Big Data, Flood Management, Open Source Software, Complex Urban Systems, Climate Change

\section{PAPER ABSTRACT / COMPLETE}

As part of the SMART Infrastructure Facility's 'GeoSocial Intelligence for Urban Livability \& Resilience' Research Group, Cls Dr. Etienne Turpin, Dr. Tomas Holderness, and Dr. Rohan Wickramasuriya, with the support of SMART IT architect Dr. Matthew Berryman and designer Sara Dean (M.Arch, MSc. Design), have developed the crowd-sourcing data collection project PetaJakarta.org (Map Jakarta). The overall aim of the project is to advance our capacity to understand and promote the resilience of cities to both extreme weather events as a result of climate change and to long-term infrastructure transformation as a process of climate adaptation. PetaJakarta.org is a pioneering webbased platform that harnesses the power of social media to gather, sort, and display information about flooding for Jakarta residents and governmental agencies in real time. The platform runs on the open source software (OSS) known as CogniCity - an OSS platform developed by the SMART Infrastructure Facility, University of Wollongongwhich allows situational information to be collected and disseminated by community members through their location 
enabled mobile devices, and optimizes infrastructure surveys and asset management for governmental actors. Equipped with scalable mapping technology for mobile devices and a critical alert service, this software enables the communication of two-way time-critical information to and from individuals and government agencies.

As the pilot study for our long term SMART research project on urban resilience and adaptation to climate change in developing nations, PetaJakarta.org radically changes real-time data collection and feedback for flood monitoring in one of most precarious delta cities of Southeast Asia. However, Petajakarta.org is powered by the highly transferable software CogniCity, which can be deployed in other megacities, in alternate languages, and for other critical urban problems pressurized by climate change. The presentation will outline our GeoSocial Intelligence Framework, which allows us to optimize citizen participation in Next Generation Decision-Support Systems that aid in the civic co-management of complex urban systems.

PetaJakarta.org works by collecting critical data about flooding and water infrastructure from existing social media networks and then inviting users to repost their messages with geo-location data to a web-based community map. Powered by our open source software, this pilot study harnesses the power of existing social media networks to provide critical, real time information about civic infrastructure and flooding. Importantly, CogniCity is not an app; it is the software component within our GeoSocial Intelligence Framework which allows data to be collected and disseminated by community members through their location-enabled mobile devices without additional plugins or programs. Within the context of the PetaJakarta.org pilot study, our GeoSocial Intelligence Framework allows us to capture critical data about environmental variables, infrastructure functionality, and individual and government responses to seasonal weather extremes, including prolonged periods of sustained precipitation, intensifying storm systems, and increasing coastal inundation due to high tides, sea level rise, and extreme land subsidence in coastal areas of the city. As this information is made available in real time to the public on our website, it is also stored for further data analysis by our SMART Research Group.

Driven by the current trend of communication interconnectivity through social media, CogniCity taps into the existing cultural fascination with social media in Jakarta, Indonesia, while also connecting to community initiatives which often occur offline. By deploying the project from both directions at once-through the software for social media filtering and feedback, and through grassroots organizations and networks - we can harness the real potential of social media by enabling critical information to be gathered and connected to the efforts of both citizen-led and government initiatives.

While the influence of social media on urban residents is well known, our development of a GeoSocial Intelligence framework that sorts, selects, and prioritizes existing social media data to enable precise, real time analysis is both unique and innovative. Other existing platforms for community mapping, such as Ushahidi, have already made possible extensive, citizen-lead data collection; CogniCity extends this logic by soliciting individuals to act as sensors in the city via existing social media networks. Within our GeoSocial Intelligence Framework, citizens as sensors provide, for the first time, comprehensive, real time coverage of infrastructure resilience throughout the city. Through our pilot study in Jakarta, this framework will allow us to study patterns of urban resilience to flooding and inundation with unprecedented detail. However, as our data analysis, public reports, and scientific findings are completed, we can also begin the design of integrated, decision-support tools for individual users, community organizations, and governmental agencies.

Our key objectives for the PetaJakarta.org Joint Pilot Study, conducted with the Jakarta Emergency Management Agency (BPBD DKI), with the support of the United Nations Pulse Lab and the Twitter \#Data Grant, can be summarized as follows:

1. Develop our understanding of urban resilience in the context of rapid urbanization and changing infrastructure demands;

2. Capture the interactions between society and urban infrastructure as a function of resilience to change;

3. Quantify probabilistic risks to urban systems as a result of environmental changes;

4. Understand the response of communities to a range of pressures on civic infrastructure;

5. Employ new data sources (i.e. crowd-sourced, social media information) to analyze 


\section{RESILIENCE PATTERNS IN SOUTHEAST AND SOUTH ASIAN MEGACITIES.}

The PetaJakarta.org pilot study develops novel techniques and methods to better understand the response of the complex urban system to change, to promote new open source technological solutions to emergent problems within complex urban systems, and to theorize and disseminate these innovative findings through scholarly publications and conference presentations. A critical component of this research is understanding the interactions between society and infrastructure in order to quantify the response of cities to a range of scenarios in a probabilistic manner. This understanding will be derived from our analysis of data collected through our GeoSocial Intelligence Framework, which uses a public, web-based interface to display crowd-sourced geo-located information.

This open framework is currently being piloted in Jakarta, Indonesia, where our main objective is studying local communities and government officials as they develop co-management strategies to promote and sustain urban resilience. By integrating a network model of the flood management infrastructure, crowd-sourced information about on-the-ground situations, and government data from flood monitoring sites throughout the city, PetaJakarta.org will operate as a public information service, a site for the dissemination of time-critical information, and a data repository that will facilitate our integrated, ongoing analysis of urban resilience. In this respect, our project reduces the redundancy in our analysis by using the same framework for data collection and for the future development of more comprehensive decision-support systems that will compliment our real time mapping and data visualization.

As cities evolve to become increasingly complex systems of people and interconnected infrastructure, the impacts of both extreme and long-term climate change are significantly heightened. Understanding the resilience of urban systems and communities in an integrated manner is key to ensure the adaptability of cities to climate change, and the sustainability of urban populations and social welfare, both of which face considerable challenges in the $21^{\text {st }}$ century. As Southeast Asia's most populous and most dense metropolitan conurbation, and the second largest urban footprint in the world, Jakarta's residents are highly exposed to rapid transformations of urban structures and systems. Recent trends in weather intensification, sea level rise, extreme pollution, severe land subsidence, and river and coastal inundation make Jakarta a key site for researching and responding to the $21^{\text {st }}$ century challenges of urban resilience and climate adaptation. Moreover, the combination of Jakarta's progressive municipal government, civil society organizations, and foreign capital investment suggest a unique potential for both transforming and improving the social life of residents through the technologically-sophisticated, scientifically-innovative, and publicly accessible networked GeoSocial Intelligence Framework.

Importantly, while Jakarta's rate of urbanization, rate of subsidence, and infrastructure systems are geographically specific, it is not the only delta city to face tremendous hardship because of climate change. Chief among the difficulties for Jakarta-as well as for Southeast Asian cities like Bangkok, Ho Chi Minh City, Manila, and Phnom Penh, as well as Dhaka in Bangladesh's Bengal Delta, and Colombo, Sri Lanka, in South Asia - is the problem of flooding. Globally, flooding causes approximately half of all human deaths due to natural disasters and accounts for nearly $40 \%$ of all natural disasters worldwide. In the developing nations of the global south, these statistics become even more dire because of failing and poorly maintained infrastructure, municipal corruption, poor intergovernmental coordination, and a lack of funds for comprehensive infrastructure improvement. In the major delta cities of South and Southeast Asia, we can add poor hydrological and hydraulic planning, frequent mismanagement of waste and sewage, difficulty in transport due to traffic extreme congestion, and extreme pollution to the list of problems exacerbating the difficultly of solving the problem of flooding. And, while the costs to insurance companies and civic governments who must bear the growing burden of post-flood reconstruction and clean up efforts continues to skyrocket, we are only beginning to understand the equally severe impact of recurrent flooding on physical and mental human health and high-risk mass migration. Combined, these factors make effective flood management the top priority for investment in South and Southeast Asian infrastructure.

However, such urgent investments are often too costly, too politically-sensitive, or simply too difficult within the existing conditions on the ground to be achieved in a timely, effective manner. Our research has demonstrated clearly that what is lacking - and what is most urgently needed-is a decision-support system that can provide accurate, real time information to municipal agencies and government actors, allowing them to make evidence-based, politically-transparent, safe and effective choices in response to extreme weather events. These cities do not need more infrastructure; they need smarter systems that can manage existing civic assets, locate weaknesses, prioritize improvements, and coordinate intergovernmental efforts to relieve our cities of costly flooding due to climate change. Until CogniCity, and our Next Generation Decision-Support System, even when they could be imagined, such 
powerful tools for infrastructure co-management were not yet available or were too cost prohibitive to be useful in a developing nations context.

Our project asks how innovative techniques for data collection can utilize the existing public enthusiasm for social media and extensive mobile communication networks to improve urban resilience in relation to flood management, weather-related emergencies, and emergency response. While addressing the problem of flooding in Jakarta is urgent, the significance of this research problem extends well beyond both Jakarta's geography and the specificity of flooding and inundation. In fact, how to better utilize the extensive network of personal mobile communication devices and social media platforms to improve urban resilience is a critical area of research for future climate adaptation and planning.

While the proliferation of social media might first appear as so much noise for civil and information system engineers, with the proper technologies for gathering, sorting, and analyzing data, this noise can be transformed into critical information for both understanding and promoting resilience. By connecting network models of urban infrastructure to crowd-sourced and social media-based data collection, and then making this information and analysis available through a web-based platform, our project links innovative areas of infrastructure research and multiplies the potential of each by producing a novel, open framework for citizen-participation in the co-monitoring and co-management of urban systems.

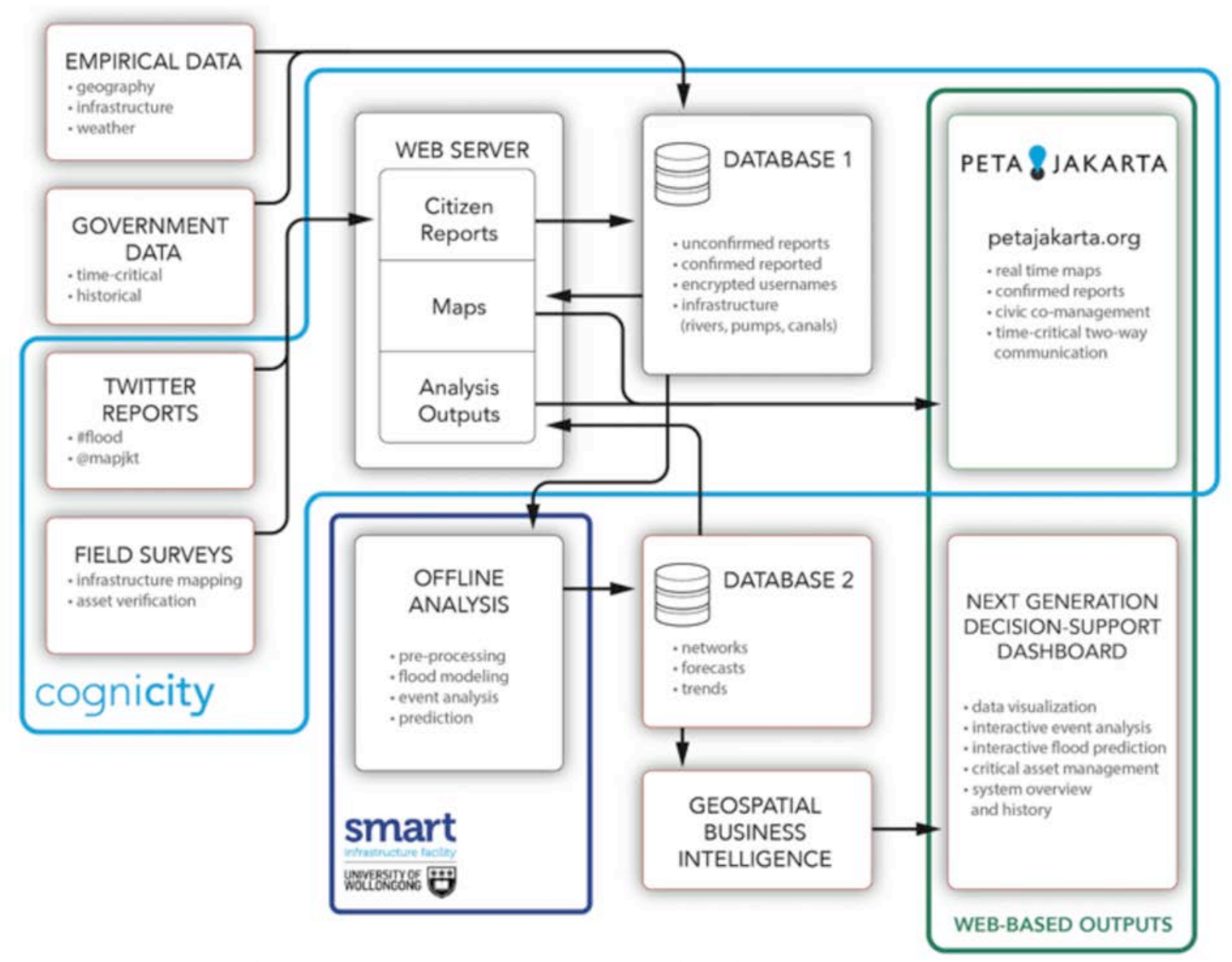

Figure 1: PetaJakarta.org System Diagram showing relation between CogniCity,

SMART Infrastructure Facility offline analysis, and public and government web-based platforms. 